\title{
GENOMIC AND EPIGENOMIC PREDICTORS FOR VARIOUS CLINICAL PHENOTYPES OF MYASTHENIA GRAVIS
}

DOI: 10.36740/WLek202103117

\author{
Elena M. Klimova', Larisa A. Drozdova', Elena V. Lavinskaya', Dmitriy V. Minukhin², Iryna O. Kudrevych', \\ Oleksandr M. Kudrevych ${ }^{4}$ \\ 'STATE INSTITUTION «ZAYCEV V.T. INSTITUTE OF GENERAL AND URGENT SURGERY OF NATIONAL ACADEMY OF MEDICAL SCIENCES OF UKRAINE», \\ KHARKIV, UKRAINE \\ ${ }^{2}$ KHARKIV NATIONAL MEDICAL UNIVERSITY, KHARKIV, UKRAINE \\ ${ }^{3}$ KHARKIV MEDICAL ACADEMY OF POSTGRADUATE EDUCATION, KHARKIV, UKRAINE \\ ${ }^{4}$ V. N. KARAZIN KHARKIV NATIONAL UNIVERSITY, KHARKIV, UKRAINE
}

\begin{abstract}
The aim: To evaluate the relationship of certain alleles of HLA class II leukocyte antigens and the profile of antibodies to various subunits of nicotinic acetylcholine receptors (nAChR), the level of Treg lymphocytes and the serum concentration of anti-inflammatory lL-10 for various clinical myasthenia gravis phenotypes.

Materials and methods: We examined 217 patients with thymus-independent myasthenia $(n=42)$ and thymus-dependent myasthenia, among them patients with thymus hyperplasia ( $n=108$ ) and thymoma ( $n=67)$. We used the following methods: ELISA, flow cytometry, light and fluorescence microscopy.

Results: Certain genomic (polymorphism of leukocyte HLA-DR antigens) and epigenomic (antibodies to a1 and a7 nAChR subunits, expression of Treg lymphocytes and concentration of cytokines) predictors were identified for various myasthenia phenotypes. The presence of HLA haplotypes DR2 and DR7 in some young patients with M with disease progression led to the development of myasthenia gravis with thymoma (MT) at an older age. The presence of a7 nAChR subunit on thymocyte mitochondria was revealed, which is an additional autoimmune target for autoantibodies in patients with myasthenia gravis. An increase in the concentration of cytokines (IL-4, IL-8, IFN- $\gamma$ ) in all patients with myasthenia gravis was revealed.

Conclusions: Estimate the features of the formation of various variants of the immune response in thymus-independent and thymus-dependent myasthenia gravis is a necessary condition for targeted immunocorrection or surgery.
\end{abstract}

KEY WORDS: myasthenia gravis, leukocyte antigens HLA-DR, nAChR, Treg-lymphocytes

Wiad Lek. 2021;74(3 p.l):475-480

\section{INTRODUCTION}

Recently, the frequency of autoimmune pathology, which includes myasthenia gravis, previously considered a rare disease, has increased many times. And now the frequency of the disease has increased 10-15 times. In myasthenia gravis, autoimmune processes are induced, the mechanisms of which affect various levels of the body from systemic to subcellular. In myasthenia gravis, autoimmune processes are induced, and their mechanisms affect various levels of the body from systemic to subcellular. Disorders of neurotransmitter reactions and progressive muscle weakness are formed in patients with myasthenia, which develop against the background of structural and functional disorders of the thymus [1]. Myasthenia gravis is clinically heterogeneous, synaptic disorders have different localization, and structural and functional changes of the thymus can manifest in the form of dysplasia, hyperplasia or thymoma $[2,3]$.

Myasthenia gravis has a multifactorial nature, depending on various etiological factors, such as genetic and epigen- etic. The debut of the disease in patients with myasthenia gravis, as a rule, corresponds to the irreversible phase of the process, when many links of the pathogenesis are formed [4]. The standard treatment for myasthenia gravis involves the use of drugs aimed at eliminating the effects of autoimmune aggression (anticholinesterase drugs, corticosteroids, plasmapheresis), which usually gives a short-term effect. Surgical removal of the thymus, as the target of autoimmune aggression, does not always allow avoiding anticholinesterase drugs, and sometimes leads to the development of myasthenic and cholinergic crises (in $20 \%$ of patients). And, as a rule, after a thymectomy, myasthenic disorders resume. The applied complex treatment of myasthenia gravis is effective only in $30-40 \%$ of patients $[5,6]$. Further progress in the treatment of myasthenia gravis is not possible without understanding the fundamental mechanisms of the development of autoimmune pathology. There is a problem of differential diagnosis and the choice of individual treatment tactic for various types of myasthenia gravis. 


\section{THE AIM}

To evaluate the relationship of certain alleles of HLA class II leukocyte antigens and the profile of antibodies to various subunits of nicotinic acetylcholine receptors ( $\mathrm{nAChR}$ ), the level of Treg lymphocytes and the serum concentration of anti-inflammatory IL-10 for various clinical myasthenia gravis phenotypes.

\section{MATERIALS AND METHODS}

We examined 217 patients with thymus-independent and thymus-dependent myasthenia (with the morphological and functional changes in the thymus - hyperplasia and thymoma), which were divided into groups depending on the clinical phenotype of this disease. The first group was consisted of 42 patients with thymus-independent myasthenia $(\mathrm{M})$, the second group included patients with myasthenia with thymus hyperplasia $(\mathrm{MH})-108$ patients, the third group - 67 patients with myasthenia and thymoma (MT).

We studied genomic (HLA-DR) and epigenomic (antibodies to $\alpha 1$ and $\alpha 7 \mathrm{nAChR}$ subunits, differentiation clusters CD45+ and CD4+CD25+, cytokine profile) factors in myasthenia gravis. The possible association of the presence of haplo- and diplotypes of leukocyte antigens of the second class HLA-DR with various clinical phenotypes of myasthenia gravis in a microlymphocytotoxic test was evaluated [7].

The determination of antibodies to the $\alpha 1$ and $\alpha 7$ subunits of $\mathrm{nAChR}$ was performed by enzyme-linked immunosorbent assay (ELISA) using the recombinant extracellular domains $\alpha 1$ (1-208) and $\alpha 7$ (1-208) as antigens [8].

The method of determining the presence of an additional autoimmune target of $\alpha 7$ subunits of $\mathrm{AChR}$ in mitochondria was performed in thymus and thymoma preparations. A cell suspension was obtained by homogenizing the organ in Hanks solution and filtering through a nylon filter. The preparation of isolated mitochondria from the thymus and thymoma was obtained by differential centrifugation. To obtain a mitochondrial precipitate, the isolation medium of the following composition was used: $10 \mathrm{mM}$ HEPES, $200 \mathrm{mM}$ sucrose, $1 \mathrm{mM}$ EDTA-1 ( $\mathrm{pH} 7.4$ ). Isolation was carried out at $2{ }^{\circ} \mathrm{C}$. Mitochondria were suspended in an incubation medium of the following composition: $10 \mathrm{mM}$ HEPES, $125 \mathrm{mM} \mathrm{KCl,} 25 \mathrm{mM} \mathrm{NaCl}, 0.1 \mathrm{mM} \mathrm{P}$ (as K-phosphate buffer; $\mathrm{pH} 7.4), 5 \mathrm{mM}$ sodium succinate $\left(22^{\circ} \mathrm{C}\right)$. The protein concentration in mitochondria was determined by the Bradford method and was $0.20 \mathrm{mg} / \mathrm{ml}$. The level of a 7 mitochondrial $\mathrm{nAChR}$ subunits in thymus preparations was determined by ELISA [8].

The expression value of the total leukocyte antigen CD45 - tyrosine-specific phosphatase on lymphocytes was determined by indirect immunofluorescence method using monoclonal antibodies ("Sorbent", Russia) labeled with FITC-stain. The cells stained by corresponding antibodies were visualized by fluorescent microscope Olympus BX53.

The regulatory lymphocyte population (Treg) was analyzed by flow cytometry (Cytomics FC500, Beckman
Coulter, USA) using monoclonal antibodies CD4-PE, CD25-FITC (Beckman Coulter, USA) [9].

Determination of the serum concentration of IL-4, IL8 , IL-10 and IFN- $\gamma$ was carried out using a test system for ELISA using monoclonal antibodies adsorbed on polystyrene plates ("Vector-Best", Ukraine). The resulting antigen-antibody complex was detected using a conjugate whose peroxidase catalyzes the cleavage of the substrate (hydrogen peroxide), causing a change in the color of the indicator. The optical density was measured at a wavelength of $450 \mathrm{~nm}$ (StatFax 3200, USA) [10].

The results were analyzed using Student's t-test. Data were presented as mean \pm standard deviation $(\mathrm{m} \pm \mathrm{SD})$, using the software package "Statistika V.6".

\section{RESULTS}

High heterogeneity of the presence of class II HLA alleles in patients with myasthenia was revealed. The incidence of the DR5 phenotype in thymus-independent myasthenia gravis was $60 \%$ and it was 2 times higher than the occurrence of the DR1 and DR2 diplotypes, and 3 times more often than the DR3, DR7, DR52 phenotypes. In thymus-dependent myasthenia gravis phenotypes DR1 and DR5 were determined with a high frequency. The DR1 phenotype was found in $60 \%$ of cases, the DR5 phenotype was found in $70 \%$ and the DR2, DR3, DR7, DR52 phenotypes were much less common (Table 1 ). In the group of patients with thymus-dependent myasthenia it was revealed the higher frequency (89\%) of a DR7 leukocyte antigen diplotype. In this group the DR2 haplotype was also detected - 38.5\%.

Among patients with the DR1 phenotype $24 \%$ of them were patients with thymus-independent myasthenia gravis, $25 \%$ were patients with thymus hyperplasia and 19\% - myasthenia gravis with thymoma. Among patients with the DR1 phenotype, there were no patients with myasthenic crisis, and only $9.5 \%$ of patients with myasthenic syndrome were detected. It can be assumed that this phenotype is more closely associated with pathomorphological changes in the thymus than with the severity of neuromuscular transmission disorders. Among patients with the DR2 phenotype $38 \%$ of them were patients with myasthenia without thymus lesions, 19\% were patients with thymoma and myasthenia gravis.

Patients with myasthenia without thymus lesions were accounted for $28 \%$ of all patients with the DR5 phenotype and $21.8 \%$ with hyperplasia. The incidence of various clinical forms of myasthenia among carriers of the DR7 phenotype is less heterogeneous, however, carriers of this phenotype are more common in myasthenia gravis with thymoma. The identified sample of carriers of the DR52 phenotype in $33.3 \%$ of cases was presented by patients with neurotransmitter disorders without damage of the thymus. Approximately $16 \%$ were patients with hyperplasia and thymoma.

The concentration of autoimmune antibodies to a 1 $\mathrm{nAChR}$ was higher than the control values in all of the examined groups; this indicator was highest in the group 
Table I. Genomic markers in patients with thymus-independent (M) and thymus-dependent myasthenia (MH, MT)

\begin{tabular}{ccc}
\hline \multicolumn{2}{c}{ The frequency of haplo- and diplotypes HLA-DR Il class in myasthenia gravis } \\
\hline Thymus-independent & Thymus-dependent myasthenia \\
\hline M & MH & MT \\
\hline HLA DR2 $-35 \%$ & HLA DR $1-60 \%$ & HLA DR2 - 38\% \\
HLA DR3 - $20 \%$ & HLA DR5 - 70\% & \\
HLA DR5 $-60 \%$ & & \\
HLA DR7 $-25 \%$ & & \\
\hline
\end{tabular}

Table II. The content of antibodies to $a 1$ and a7 subunits of $n A C h R$ in patients with various clinical phenotypes of myasthenia gravis

\begin{tabular}{lccccc} 
& Control group & $\begin{array}{c}\text { Thymus-independent } \\
\text { myasthenia (M) }\end{array}$ & \multicolumn{2}{c}{ Thymus-dependent myasthenia } \\
\cline { 4 - 6 } & & Thymus hyperplasia (MH) & Thymoma (MT) \\
\hline Antibodies to a1 nAChR, units OD & $0.180 \pm 0.43$ & $0.373 \pm 0.052$ & $0.389 \pm 0.065$ & $0.281 \pm 0.027$ \\
\hline Antibodies to a7 nAChR, units OD & $0.180 \pm 0.37$ & $0.343 \pm 0.089$ & $0.268 \pm 0.034$ & $0.198 \pm 0.021$ \\
\hline
\end{tabular}

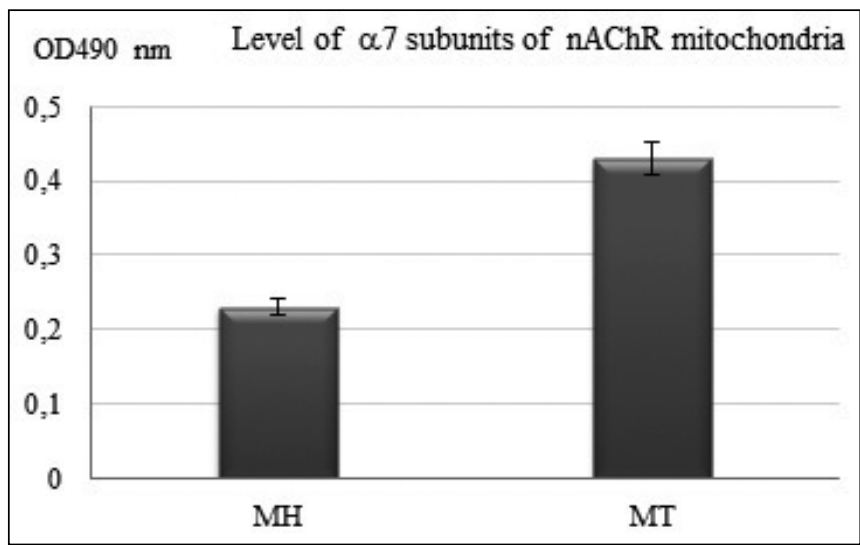

Fig. 1. Change in the level of a7-subunits of $n A C h R$ of mitochondria in thymus preparations of patients without (MH) and with thymoma (MT)

of patients with myasthenia gravis and thymus hyperplasia $(0.389 \pm 0.065$ units OD). The content of antibodies to $a 7 \mathrm{nAChR}$ was increased in groups of patients with myasthenia gravis without morphological and functional changes of the thymus and with myasthenia gravis on the background of thymic hyperplasia (Table 2).

The investigation of the characteristics of thymus cells, the central organ of immunity, will allow us to study some mechanisms of the central self-tolerance loss. As is known, nicotinic acetylcholine receptors (nAChR), related to the superfamily of ligand-dependent ion channels, are localized in synapses and ganglia of the central nervous system. These receptors containing the $\alpha 7$ subunit regulate apoptosis initiation processes by influencing voltage-dependent ion channels [11].

In patients with myasthenia gravis after thymectomy and thymomectomy ( $\mathrm{MH}$ and MT), thymus and thymoma cells revealed the presence of $\alpha 7$ subunits of $n A C h R$, which are expressed on the outer mitochondrial membrane. The level of $\alpha 7$ subunits of $\mathrm{nAChR}$ of mitochondria in the thymus in patients with thymus hyperplasia $(\mathrm{MH})$ was minimal and amounted to 0.23 units E. The maximum level of $\alpha 7$ subunits of $\mathrm{nAChR}$ on mitochondria was detected in thymus preparations with thymomas (MT group) - 0.43 units E., which was in 1.9 times higher than in mitochondria of the thymus (Fig. 1).

The localization of the $\alpha 7$ subunit of $\mathrm{nAChR}$ in mitochondria may be an additional target in the development of the processes of central self-tolerance loss in the thymus.

Inhibition of CD45+ expression on immunocompetent cells showed a decrease in the expression of total leukocyte antigen in all patients with myasthenia gravis (Fig 2 A, B, C).

The minimum level $(80.0 \pm 3.4 \%)$ of expression of the $\mathrm{CD} 45$ transmembrane protein was revealed in the group with thymus-independent myasthenia M (Fig. 2A, 3), which, in turn, is associated with a decrease in the degree of signal transmission from antigenic lymphocyte receptors to the cell core for transcription induction.

In the regulation of immunity, in particular in the induction of immune suppression, the main role is played by regulatory $\mathrm{CD} 4+\mathrm{CD} 25+\mathrm{T}$ lymphocytes. The insufficiency of the function of Treg cells is of great importance in the regulation of control during the development and progression of autoimmune diseases, in the process of oncogenesis, and in the regulation of immunity in infections [12], and also the function of CD4+CD25+ cells in autoimmune myasthenia is important. In this study, all patients showed a decrease in the expression of Treg cells. In the MH group, the level of cells with the CD4+CD25+ phenotype was on average $30 \%$ lower than in the control group. Probably, in the autoimmune myasthenia group with thymus hyperplasia (MH), CD4+ T cells with high expression of CD25 molecules prevailed to inhibit autoimmune processes, since such cells have suppressor activity (Fig. 4). In groups M and MT, a 4 -fold decrease in the expression of CD25+ on $\mathrm{CD} 4+\mathrm{T}$ cells was revealed in comparison with the control. These data indicate the presence of similar mechanisms of self-tolerance loss in these groups. Regulatory $\mathrm{T}$ lymphocytes CD4+CD25+ are a source of IL-10, which regulates autoimmune reactions. A decrease in the expression of Treg lymphocytes leads to a decrease in the synthesis of IL-10, which was noted in groups M and MT. 

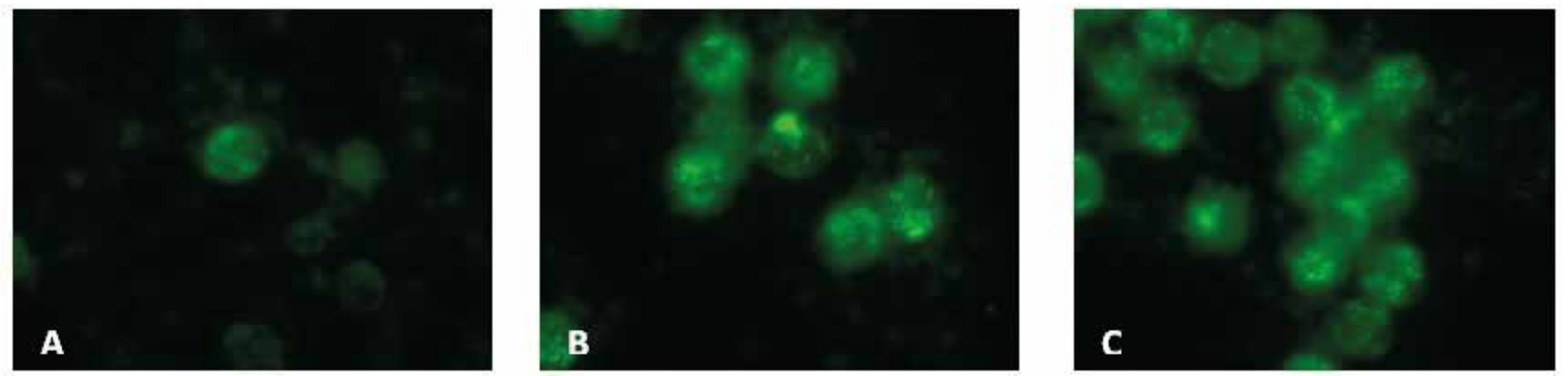

Fig. 2. Expression of CD45+ in patients with thymus-independent and thymus-dependent myasthenia gravis, stained with FITC, $x 1000: A-g r o u p ~ M$, $87 \%$; B - group MH, 91\%; C - group MT, 92\%

In all examined patients with myasthenia, an increasing the levels of IL-4, IL-8, and IFN- $\gamma$ relative to the control was revealed. The maximum value of IL- 4 was found in patients with thymomas - $(666.4 \pm 44.5) \mathrm{mmol} / \mathrm{l}$. With thymus hyperplasia against the background of myasthenia gravis, a multiple increasing the content of IL-4 was also revealed - (492.5 \pm $52.8) \mathrm{mmol} / \mathrm{l}$ (Table 3). An increasing the level of serum IL-4 concentration accompanies the development of anti-infection immunity according to the type of Th2 dependent antibody formation, since activated IL-4 Th2 lymphocytes control the development of a humoral immune response.

The maximum increasing of IL- 8 was detected in patients with myasthenia gravis, which was accompanied by a tumor of the thymus, since an increasing of this cytokine is often associated with the induction of oncogenesis, and it stimulates the release of active oxygen radicals and other bioaggressive substances. The content of IFN- $\gamma$ was increased in all groups, and the maximum increase (15 times) was revealed in the MH group (Table 3 ). Therefore, in patients of the $\mathrm{MH}$ group, differentiation along the Th1 pathway prevails.

\section{DISCUSSION}

The relationship between the clinical phenotypes of myasthenia gravis and the variants of HLA leukocyte anti-

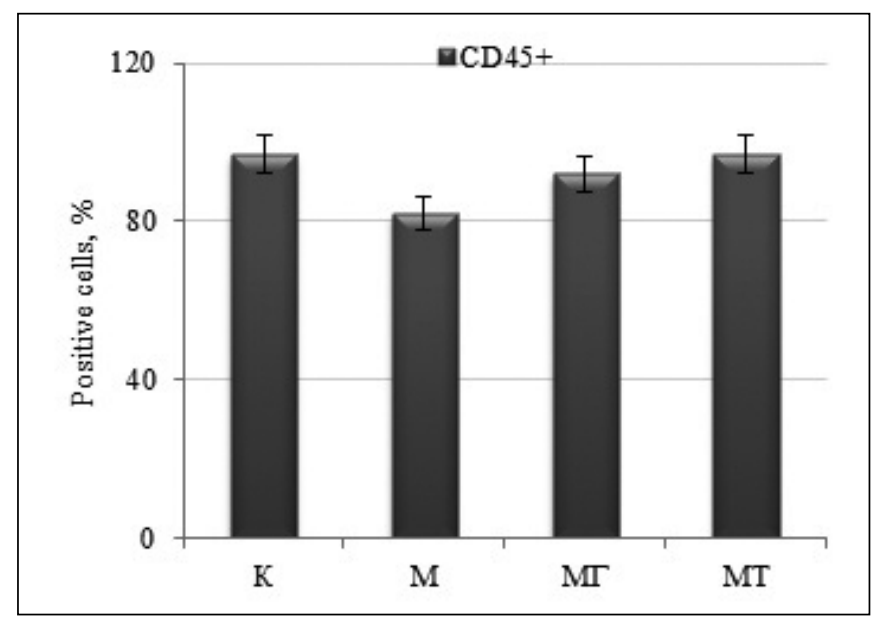

Fig. 3. Expression of $C D 45+(\%)$ in patients with various clinical phenotypes of myasthenia gravis gens, which are genetic factors that form various types of immune reactivity and determine resistance vectors, has been revealed. The characteristic associations of leukocyte antigens in $\mathrm{M}, \mathrm{MH}$ and $\mathrm{MT}$ can be used as prognostic markers for the development of types of immune imbalance that develops during the latent period of the formation of autoimmune pathology or during remission. The presence of HLA haplotypes DR2 and DR7 in some young patients with $\mathrm{M}$ with disease progression led to the development of myasthenia gravis with thymoma (MT) in the elderly.

The maximum increasing of the concentration of antibodies to the $\alpha 1$ and $\alpha 7$ subunits of $\mathrm{AChR}$ was found in patients with $\mathrm{M}$ and $\mathrm{MH}$. In patients with thymoma the concentration of specific antibodies to different domains of nAChR did not significantly differ from the control values. Therefore, in patients with MT, the pathogenetic factor of the development of myasthenic syndrome and crisis may have other mechanisms that are not associated with the presence of antibodies to nAChR.

According to Hurst et al. (2013) the function of mitochondrial $\mathrm{nAChR}$ is to control the formation of a mitochondrial pore of transitional conductivity, which is a source of proapoptotic factors and reactive oxygen species released in the cytosol [11]. In additional, the level of $\alpha 7$ subunits of nAChR is higher in the thymus of patients with thymoma, therefore, tumor transformation is accompanied

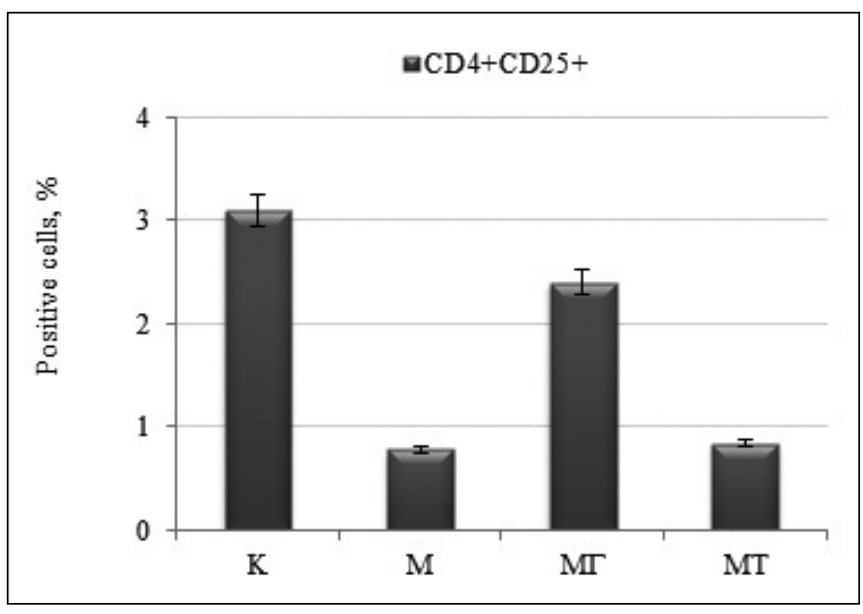

Fig. 4. The level of a subpopulation CD4+CD25+ Treg-lymphocytes in patients with various clinical phenotypes of myasthenia gravis 
Table III. Change in the level of interleukins in patients with thymus-independent (M) and thymus-dependent myasthenia gravis (MH, MT)

\begin{tabular}{ccccc}
\hline Index & Control group & M & MH & MT \\
\hline $\mathrm{IL}-4, \mathrm{mmol} / \mathrm{l}$ & $70.0 \pm 22.9$ & $337.3 \pm 59.4$ & $492.5 \pm 52.8$ & $666.4 \pm 44.5$ \\
\hline $\mathrm{IL}-8, \mathrm{pg} / \mathrm{ml}$ & $10.0 \pm 8.4$ & $72.3 \pm 21.5$ & $88.3 \pm 34.2$ & $590.9 \pm 67.1$ \\
\hline $\mathrm{IFN}-\gamma, \mathrm{pg} / \mathrm{ml}$ & $9.4 \pm 2.3$ & $67.1 \pm 10.4$ & $147.0 \pm 27.3$ & $48.5 \pm 5.3$ \\
\hline
\end{tabular}

by an increase in mitochondrial nAChR, which supports the viability of tumor cells.

It is known that the regulatory function of T lymphocytes is to control the strength and duration of the immune response by regulating the ratio of helper and cytotoxic $\mathrm{T}$ cells. Activation of $\mathrm{T}$ lymphocytes in patients with $\mathrm{M}$, which was estimated by the expression of the main leukocyte antigen of the CD45+, was the lowest in comparison with the value of this indicator in MH and MT. Membrane-binding phosphatase CD $45+$ can act as a diagnostic criterion for the development of immune imbalance in the presence of acute infection.

The regulatory population of $\mathrm{T}$ cells plays an important role in suppressing immune hyperactive reactions and prevents autoimmune and allergic diseases, but at the same time they can reduce anti-infection and antitumor immunity. Treg-cells can play, on the one hand, a protective role, limiting the development of the inflammatory process, and on the other, an increase in the number of Treg lymphocytes can lead to an increased risk of concomitant infections as a result of a decrease in the proliferative and functional activity of effector cells, which was observed in patient with thymus hyperplasia $(\mathrm{MH})$. That is, there is a change in the functional state of regulatory $\mathrm{T}$ lymphocytes, which, in turn, leads to a change in the body's resistance to infections. In the literature, there is a tendency for an increase in subpopulations of $\mathrm{CD} 4+\mathrm{CD} 25+$ cells as the disease progresses $[13,14]$. In our studies, this tendency is observed in the $\mathrm{MH}$ group, where the level of $\mathrm{CD} 4+\mathrm{CD} 25+$ is 3 times higher than in the M and MT groups. At the same time, in patients with thymus-independent (M) and thymus-dependent (MT) myasthenia gravis, we did not reveal sufficient expression of $\mathrm{CD} 4+\mathrm{CD} 25+$. But, since autoimmune diseases are accompanied by impaired immunological tolerance, in the formation of which a special role belongs to the population of Treg cells, therefore, probably in the M and MT groups, the insufficiency of regulatory cells is primarily associated with the pathogenesis of these myasthenia phenotypes.

Regulatory cytokines can take part in the self-maintenance of the pathological process and determine the strategy for the formation of a specific metabolic pattern. A change in the concentration of cytokines, along with other lysing cytotoxic factors, can lead to the formation of autoimmune conditions. A subpopulation of Th2 lymphocytes produces IL-4, which is significantly increased (i.e., it is an indirect autoimmunization factor) in patients with MH and MT. Patients with MT revealed a sixty-fold increase in the concentration of IL-8, which is a marker of oncogenesis. Obviously, an increase in the concentration of IL-8 plays a pathogenetic role in the development of the pathology of synaptic transmission and morpho-structural changes in the thymus, and this leads to the formation of locally distributed thymus.

\section{CONCLUSIONS}

Certain genomic (polymorphism of leukocyte HLA-DR antigens) and epigenomic (antibodies to $\alpha 1$ and $\alpha 7$ subunits nAChR, expression of Treg lymphocytes and concentration of cytokines) predictors were identified for various myasthenia gravis phenotypes. Evaluation of the features of the formation of various variants of the immune response in thymus-independent $(\mathrm{M})$ and thymus-dependent $(\mathrm{MH}, \mathrm{MT})$ myasthenia gravis is necessary for targeted correction of metabolic and immunological disorders. An analysis of changes in specific biomarkers, taking into account the genomic component, will allow for reasonable targeted immunocorrection or surgery.

\section{REFERENCES}

1. Priola A.M., Priola S.M. Imaging of thymus in myasthenia gravis: from thymic hyperplasia to thymic tumor. Clinical Radiology. 2014;69(5):e230-e245.

2. Berrih-Aknin S. Myasthenia Gravis: paradox versus paradigm in autoimmunity. Journal of Autoimmunity. 2014;52:1-28.

3. Klimova E.M., Lavinskaya E.V., Minukhin D.V. et al. On forming central and peripheral markers of self-tolerance loss in diverse clinical myasthenic phenotypes. Der Pharmacia Lettre. 2017; 9(6):8-17.

4. Bojko V.V., Klimova E.M., Kudrevich A.N. Lechenie miastenii s uchetom immunofiziologicheskih fenotipov. [Treatment of myasthenia gravis taking into account immunophysiological phenotypes]. Kharkov: Publishing House Sheinina E.V. 2008: 424. (in Russian).

5. NazarbaghiS.,Amiri-Nikpour M.R.,Mahmodlou R.etal.Clinical outcomes of myasthenia gravis with thymoma and thymic hyperplasia undergoing extended transsternal thymectomy: a single-center experience. North American Journal of medical sciences. 2015;7(11):503-508.

6. Klimova E.M., Bozhkov A.l., Boyko V.V. et al. Endogenic cytotoxic compounds and formation of the clinic forms of myasthenia. Translational Biomedicine. 2016;7,3,84:1-16.

7. Schreuder G.M.Th. The HLA dictionary 2004: a summary of HLA-A, $-B,-C$, $-D R B 1 / 3 / 4 / 5$ and $-D Q B 1$ alleles and their association with serologically defined HLA-A, $-B,-C,-D R$ and $-D Q$ antigens. International journal of immunogenetics. 2005;32(1).

8. Gergalova G.L., Lehmus 0.J., Skok M.V. Possible effect of activation of a7-nicotinic acetylcholine receptors in the mitochondrial membrane on the development of apoptosis. Neurophysiology. 2011;43(3):195-197.

9. ParcsD.R., LanierL., Herrengerg L.A. Flow cytometryand fluorescenseactivated cell sortins (Facs). Handbook of Experimental Immunology. 1986;302-324.

10. De La Rica R., Stevens M.M. Plasmonic ELISA for the ultrasensitive detection of disease biomarkers with the naked eye. Nature Nanotechnology. 2012;7(12),12:821. 
11. Hurst R., Rollema H., Bertrand D. Nicotinic acetylcholine receptors: from basic science to therapeutics. Pharmacology therapy. 2013;137(1):22-54.

12. Sadeghifard N.T., Talaei M.H., Naser A. et al. Maleki regulatory cell and body tolerance. Der Pharmacia Lettre. 2017;9(5):23-28.

13. Fattorossi A., Battaglia A., Buzzonetti A. et al. Circulating and thymic CD4+CD25+ T regulatory cells in myasthenia gravis: effect of immunosuppressive treatment. Immunology. 2005;116:134-141.

14. Balandina A., Lecart S., Dartevelle P. et al. Functional defect of regulatory CD4(+)CD25+ T cells in the thymus of patients with autoimmune myasthenia gravis. Blood. 2005;105:735-741.

We are grateful to Professor Skok M.V. and Ph.D. Lykhmus $O$. Yu. for assistance in research on the detection of antibodies to the $\alpha 1$ and $\alpha 7$ subunits of $n A C h R$ and $\alpha 7 n A C h R$ of the thymus mitochondria carried out in the Laboratory of Cellular Receptor Immunology of the Palladin Institute of Biochemistry of the National Academy of Sciences of Ukraine. The work was carried out in accordance with the research plan of State institution "Zaycev V.T. Institute of general and urgent surgery of National academy of medical sciences of Ukraine and Kharkiv National Medical University in 20162018, research work "A study of the self-tolerance regulation of circulating immunocompetent cells and thymocytes in patients with myasthenia gravis».

\section{ORCID and contributionship:}

Elena M. Klimova: 0000-0002-4007-6806 ${ }^{A, D, E}$

Larisa A. Drozdova: 0000-0001-9678-4046 ${ }^{A, B, D, E}$

Elena V. Lavinskaya: 0000-0001-5813-3656 C,D,E,F

Dmitriy V. Minukhin: 0000-0003-3371-1178 ${ }^{\text {B,E,F }}$

Iryna O. Kudrevych: 0000-0002-6778-3498 C,F

Oleksandr M. Kudrevych: 0000-0002-2086-8822 ${ }^{B, F}$

\section{Conflict of interest:}

The Authors declare no conflict of interest.

\section{CORRESPONDING AUTHOR}

\section{Elena M. Klimova}

Zaycev V.T. Institute of general and urgent surgery of National academy of medical sciences of Ukraine 1, Balakireva vyizd, 61103, Kharkiv, Ukraine tel: +38(057)3494115

e-mail:klimovalena53@gmail.com

Received: 16.04 .2020

Accepted: 11.11 .2020

A - Work concept and design, B - Data collection and analysis, C - Responsibility for statistical analysis,

D-Writing the article, $\mathbf{E}$-Critical review, $\mathbf{F}$ - Final approval of the article 